\section{$\underset{\substack{\text { hommes } \\ \text { \& migrations }}}{ }$}

\section{Hommes \& migrations}

Revue française de référence sur les dynamiques

migratoires

$1304 \mid 2013$

Frontières

\title{
Éléments de construction de la territorialité en
}

France

Entre objectif politique et expérience individuelle et artistique

\section{Patrick Picouet}

\section{(2) OpenEdition}

Journals

Édition électronique

URL : http://journals.openedition.org/hommesmigrations/2673

DOI : $10.4000 /$ hommesmigrations. 2673

ISSN : 2262-3353

Éditeur

Musée national de l'histoire de l'immigration

Édition imprimée

Date de publication : 1 octobre 2013

Pagination : 140-144

ISBN : 978-2-919040-24-7

ISSN : 1142-852X

Référence électronique

Patrick Picouet, «Éléments de construction de la territorialité en France », Hommes \& migrations [En ligne], 1304 | 2013, mis en ligne le 17 mars 2014, consulté le 22 septembre 2020. URL : http:// journals.openedition.org/hommesmigrations/2673; DOI : https://doi.org/10.4000/ hommesmigrations.2673 


\section{ÉLÉMENTS DE CONSTRUCTION DE LA TERRITORIALITÉ EN FRANCE \\ ENTRE OBJECTIF POLITIQUE ET EXPÉRIENCE INDIVIDUELLE ET ARTISTIQUE}

PHILIPPE PICOUET, géographe, professeur des universités, université de Lille-I

Pour la société, et pour chaque individu, la relation au territoire (territorialité) et à la frontière (frontiéralité) s'appuie largement sur les cartes où les limites sont définies par des lignes. Cellesci deviennent des icônes de la frontière pour la plupart des citoyens. Les manuels scolaires de nombreux pays sans doute et, en particulier, les manuels de géographie de la France, véhiculent ces images pendant tout le XXe siècle.

À côté de ces manuels exprimant les points de vue des programmes imposés par l'État, des artistes français proposent d'autres regards et d'autres relations à la frontière. Ils façonnent, selon les circonstances et selon l'engagement des auteurs, des images sensiblement différentes. En partant de quelques exemples, de différentes échelles et temporalités, nous pouvons souligner les écarts entre la logique structurelle de la géographie enseignée et la logique relationnelle de l'expérience individuelle et artistique.

\section{Les manuels scolaires et l'image de la ligne frontière}

Au début de la III République, la géographie et les manuels scolaires de géographie servent, comme discipline et outil, à soutenir le régime politique fragilisé par les visées monarchistes et par l'amputation de son territoire. La géographie coloniale et la géographie politique structurent la discipline et occupent une place non négligeable dans les manuels. L'étude des frontières françaises permet aux jeunes Français de bien connaître le territoire national et en particulier ses lignes, éléments naturels, bastions, et places fortes en organisant la défense. Dans Le Tour de France par deux enfants', André et Julien tracent les limites du pays et évoquent avec émotion la vie de ces Alsaciens séparés de la France audelà de la ligne bleue des Vosges. Les cartes des manuels et atlas de l'époque font des frontières françaises une véritable ligne de front annonçant les combats futurs (fig. 1).

Durant quasiment tout le XXe siècle, les manuels scolaires véhiculent des images de frontières souvent décalées par rapport à la réalité. Les frontières naturelles, le caractère inquiétant des frontières du Nord constituent une trame lexicale et cartographique toujours représentée. La frontiéralité transmise dans les manuels scolaires correspond à une logique nationale de formation des soldats, d'éducation des citoyens et à une iconographie de la résistance aux changements (dans le sens de Jean Gottmann²). La frontière est étudiée d'un point de vue instru- 


\section{L'image d'un entre-deux}

À plus grande échelle, la frontière se dilate. Maxence van der Meersch publie en 1932 un premier roman, La Maison dans la dune, qui le situe d'emblée, aux yeux des critiques, dans la lignée des grands écrivains naturalistes tels que Zola. II y raconte l'histoire tragique de contrebandiers, dont Sylvain le héros au grand cœur, qui a abandonné une carrière prometteuse de boxeur pour épouser une ancienne prostituée. Voulant satisfaire les goûts dispendieux de sa femme, il suit son ami César sur les sentiers transfrontaliers surveillés par Lourges, l'un des plus redoutables douaniers. Entre Furnes et Dunkerque, l'auteur décrit "le littoral dans sa partie désertique, sablonneuse, qui s'étend, toute nue, aride et presque inculte, sur des kilomètres et des kilomètres3". Entre la discontinuité du littoral, avec ses dunes mouvantes et la frontière franco-belge, la plaine est morne, abandonnée au "vent dur et salin". Les paysages des dunes et des Moëres donnent une touche de mystère et de rudesse à l'histoire.

Atlas Levasseur, 1874

La frontière devient un espace de vie, certes difficile mais où un entre-deux existe ; le long

mental et la ligne la représente sur les cartes en général et celles des manuels scolaires en particulier. Tardivement évoqué mais très rapidement exagéré, l'effacement des frontières souligne plus une volonté politique qu'une réalité vécue et acceptée. À côté des manuels scolaires de géographie où s'expriment les points de vue des programmes imposés par l'État, les artistes français proposent d'autres regards et d'autres relations à la frontière. Trois exemples, un roman, des mémoires et un film, nous apportent des éléments de réflexion dans le contexte des années 1930 et des années 1950-1960. du canal de Furnes à Dunkerque, Sylvain rencontre par hasard une petite auberge, perdue en bordure d'une route abandonnée, habitée par des vieillards et une très jeune fille, Pascaline. Ce lieu et ses habitants le séduisent. À l'écart du monde, un endroit caché par une végétation exubérante, c'est un peu un paradis rêvé pour lui, il y redevient enfant, un adolescent avec Pascaline. II s'investit beaucoup dans les travaux d'entretien; il imagine une nouvelle vie possible. Dans cet espace secret, il peut revivre et se racheter, abandonner sa vie de contrebandier du tabac. C'est là qu'il vient finalement mourir. 


\section{REPÉRAGE}

La ligne, frontière de référence dans les manuels scolaires, est presque effacée dans le roman qui donne une épaisseur à la frontière, espace d'une autre vie possible et des échanges illégaux de la contrebande. Nous pouvons mesurer à quel point l'écart est grand entre les préoccupations nationales dans le contexte menaçant des années 1930 et l'expression de la vie quotidienne des habitants de la frontière, entre Furnes et Dunkerque.

\section{L'image d'une société transfrontalière}

Nous retrouvons cette vie frontalière à la fois dangereuse et solidaire dans l'histoire de la contrebande du nord de la France. Dominique Roger évoque les souvenirs des "passeurs du clair de lune" dessinant un espace de jeu nocturne de part et d'autre de la frontière ${ }^{4}$. Les enfants fréquentent les mêmesécoles, certains deviennent douaniers, d'autres fraudeurs. Très tôt, ils apprennent tous les arcanes du métier grâce à leur père ou grand-père ; d'abord

Durant quasiment tout le XXe siècle, les manuels scolaires véhiculent des images de frontières souvent décalées par rapport à la réalité. guetteurs, adultes ils deviennent passeurs.

D'autres jouent avec la ligne tel celui qui construit son cabaret à cheval sur la frontière. "La salle de bal est en France et les cuisines en Belgique. La marchandise arrive directement d'Anvers, passe devant les fourneaux, puis elle sort en zigzaguant entre les danseurs... II suffit de faire glisser les sacs sur dix mètres pour amasser une fortune ${ }^{5}$ !"

Dominique Roger nous montre une société de la frontière qui se reconnaît dans un jeu transfrontalier, avec ses héros, ses profiteurs et sa souffrance. Dès l'enfance, les règles en sont connues; pour beaucoup, sans doute, l'amusement de l'enfant n'est jamais loin, comme le souligne avec humour Erik Orsenna : "Les douaniers s'amusaient autant que nous, demandez-leur si vous ne me croyez pas. Maintenant, ils s'ennuient pareil. Ah ! La mort des frontières n'a pas fait que des heureux ${ }^{6}$ !"

À partir des années 1870 et jusqu'en 1950, en un temps durant lequel se cristallisent les nationalismes et les idéologies, existe un véritable territoire économique : le travail transfrontalier de la Belgique vers la France est alors très important, de même que la contrebande. C'est aussi un territoire culturel dans lequel se reconnaissent et se marient des habitants de part et d'autre de la ligne frontière. La discordance entre les temporalités politiques et les temporalités socioculturelles est remarquable sur la frontière franco-belge à cette époque.

\section{Dans les montagnes des Alpes du Sud : l'image d'une ligne concrète mais mouvante}

Vue à grande échelle, dans la montagne de l'arrière-pays niçois et plus précisément dans le village d'Assola, la ligne frontière perd de son origine naturelle mais concrétise la délimitation précise des nations française et italienne. Le film de Christian Jaque, La loi c'est la loir, présente de ce point de vue le plus grand intérêt. Dans cette comédie tournée au lendemain de la Deuxième Guerre mondiale, à une époque, sans doute, durant laquelle le sujet pouvait prêter à sourire, l'auteur aborde le tracé, les fluctuations de la ligne et leurs conséquences sur la vie quotidienne des habitants d'une petite ville de la fron- 
tière. Le film montre le "spectacle" de la frontière avec ses images connues de tous : d'abord, la ligne en pointillés qui traverse les Alpes méditerranéennes et le village d'Assola, dans un tracé particulièrement sinueux et apparemment arbitraire, recoupant une rue dans le sens longitudinal (aux revêtements très visiblement différents de part et d'autre) et même l'auberge dont le bâtiment est français d'un côté et italien de l'autre ; puis le poste de douane, la police de la frontière, le pont coupé, peut-être une trace d'un ancien conflit; enfin, de l'autre côté, la cuisine italienne ! La nation française se reconnaît d'autant plus que les premières scènes du film se déroulent le 14 juillet, avec un défilé qui longe la ligne sur l'air de Sambre et Meuse! Les premiers spectateurs sont les Italiens de l'autre côté de la rue!

Les territoires deviennent plus flous lorsque la frontière se déplace, avec ses conséquences humaines, jusqu'aux plus imprévisibles : le douanier français, représentant de la loi par excellence soudain obligé de quitter la France, est accusé de désertion par les uns puis considéré comme apatride par les autres. Le contrebandier italien, son pire ennemi, lui, le passeur, le seul qui s'exprime dans les deux langues, le sortira d'affaire. Le spectacle des nations se brouille, les différences sont moins nettes. 1957, l'année de la sortie du film qui développe l'idée de la dynamique de la frontière est aussi celle de la signature du traité fondateur de la CEE.

Si l'on considère comme Benedict Anderson que la nation est une communauté politique imaginaire (parce que les citoyens ne se connaissent pas), et imaginée comme intrinsèquement limitée et souveraine (même la plus grande a des frontières finies) ${ }^{8}$, incontestablement le système éducatif, et plus particulièrement les manuels scolaires, ont contribué durablement à instiller une idéologie nationale dans la société.

Depuis la fin du XIXe siècle, la frontière occupe une place de choix, quoique à éclipse, dans les images de la nation française. Durant les années 1870, elle est une frontière étroitement associée 


\section{REPÉRAGE}

aux reliefs, fleuves et mers ou océans, et une ligne de front articulée aux places fortes de l'arrière, dont les élèves doivent connaître tous les éléments. Pendant tout le $X X^{e}$ siècle, elle reste une ligne dont le tracé définit une figure géométrique aisément reconnaissable, mais aussi une figure harmonieuse et équilibrée, presque idéale, I'hexagone. Celui-ci devient finalement un logo utilisé par les hommes politiques et les publicitaires. Benedict Anderson a bien repéré cette évolution dans les pays coloniaux en évoquant la "logoïsation générale" dans les manuels scolaires, etc. De ce logo, les géographes font même un modèle graphique ${ }^{10}$ (fig. 2).

En 1957, sur la frontière franco-italienne, Christian Jaque dessine concrètement la ligne, celle qui ne suit pas les reliefs et celle qui traverse des petites villes. Elle est un miroir dans lequel deux sociétés se regardent, et une ligne de part et d'autre de laquelle deux souverainetés s'exercent, avec les aléas des variations du tracé et leurs conséquences individuelles.

Sans doute les représentations transmises par les manuels scolaires durant les années de formation des futurs citoyens s'imposent-elles durablement dans les pensées. Le contexte historique et politique contribue souvent à conforter les images apprises : dans les années 1930 comme dans les années 1950, le territoire national (son contrôle et sa protection) et l'unité de la société comptent plus que les pratiques et les sentiments des individus. Dans les manuels scolaires, la cartographie joue un rôle majeur dans l'imprégnation des esprits; nous l'avons vu, l'image de la France, I'hexagone, devient un symbole important. Nous pouvons nous demander ce qu'il en est, lorsqu'un nouveau territoire tente de se former comme I'Union européenne actuellement. . 